\title{
Edge-Preserving Denoising for Segmentation in CT-Images
}

\author{
Eva Eibenberger, Anja Borsdorf, Andreas Wimmer, Joachim Hornegger \\ Lehrstuhl für Mustererkennung, Friedrich-Alexander-Universität Erlangen-Nürnberg \\ anja.borsdorf@informatik. uni-erlangen.de
}

\begin{abstract}
In the clinical environment the segmentation of organs is an increasingly important application and used, for example, to restrict the perfusion analysis to a certain organ. In order to automate the time-consuming segmentation process denoising techniques are required, which can simultaneously remove the locally varying and oriented noise in computed tomography (CT) images and preserve edges of relevant structures. We analyze the suitability of different edge-preserving noise reduction methods to be used as a pre-processing step for Geodesic Active Contours (GAC) segmentation. Two popular methods, bilateral filtering and anisotropic diffusion, are compared to a wavelet-based approach, which is adjusted to the CT-specific noise characteristics. We show that robust segmentation results for different organs at varying noise levels can only be achieved using the wavelet-based denoising. Furthermore, the optimal selection of parameters for the bilateral filter and the anisotropic diffusion is highly dependent on the dataset and the segmentation task.
\end{abstract}

\section{Introduction}

In computed tomography $(\mathrm{CT})$, the projections acquired at the detector are corrupted by quantum noise. This noise propagates through the reconstruction to the final volume slices. There, noise is non-stationary, its distribution is unknown and directed noise can be present due to high attenuation along certain directions.

In order to improve the reliability of automatic segmentation algorithms, noise has to be removed. The most basic approach is to apply a linear Gaussian filter. The main drawback of low pass filtering is that important image details like edges are smoothed, too. As neighboring structures often show similar intensities, the segmentation may leak into these regions. This effect can be avoided or at least reduced by using edge-preserving noise reduction methods. In this paper the suitability of different denoising approaches as a pre-processing step for edgebased segmentation of organs in CT-images is investigated. Two standard edgepreserving filters are compared to a wavelet-based approach, which adapts itself to the characteristics of noise in $\mathrm{CT}$. 


\section{Methods}

The image data used for the evaluation consists of 2D CT-slices with different amplitudes of noise. The segmentation is performed for the liver, the kidney and the spleen. Certain features, e.g. strong, non-stationary noise, changing contrasts at the boundary, small structures due to contrast agent or diffuse boundaries make the selection of optimal parameters for the denoising and segmentation algorithm difficult. For the clinical practice, methods are useless where too many parameters must be adjusted to the specific problem or dataset.

The edge-based GAC approach [1] is used for segmentation. The parameters for the GAC were optimized once ([propagation, curvature, advection] $\in$ $[(1,1,3),(1,2,4)])$ and then left unchanged, because here the influence of the pre-processing should be investigated. The considered noise reduction methods are: the bilateral filtering [2], the anisotropic diffusion [3] and a combination of two wavelet approaches based on thresholding [4] and correlation analysis [5]. Several parameters can be adjusted for the examined denoising methods. For the bilateral filtering, $\sigma_{D}$ and $\sigma_{R}$, the standard deviations for the image domain and image range, as well as the mask size $m$ have to be specified. The anisotropic diffusion requires the number of iterations $N_{\text {iter }}$ and the contrast parameter $\lambda$. For the wavelet-based method, the mode of thresholding (hard or soft), a factor $k$ specifying the amount of thresholding and a parameter $N$ defining the amount of noise suppression of the correlation analysis have to be selected.

For each filter the parameter combination resulting in the best segmentation was determined for each organ in the medical data. We used the manual segmentation of a medical expert as reference. Afterwards, the results were analyzed on the basis of simulated water-phantoms with inlaid objects of different contrasts and with varying noise amplitudes. The quality of the segmentation result was quantified through sensitivity and specificity. During the evaluation, especially the robustness with respect to parameter variations, changing organs and varying noise amplitudes were examined.

\section{Results}

For each organ in both datasets and all filtering methods, it was possible to find segmentation contours almost perfectly fitting the manual segmentation in Fig. 1(a). However, some important differences could be observed with respect to the robustness of the segmentation result regarding the parameter selection of the denoising. These differences are now described on the example of one segmented liver. Similar results were observable for all other investigated organs.

Figure 1(e) shows the best liver segmentation for the bilateral filtering. If the contrast-dependent $\sigma_{R}$ was increased, the segmentation contour leaked at the region near the kidney (Fig. 1(f)), as the boundary is slightly diffuse and of low contrast here. It could be observed that for higher noise amplitudes the sensitivity to parameter variations was intensified. The best segmented liver for the anisotropic diffusion is shown in Fig. 1(b). Both Fig. 1(c) and Fig. 1(d) illustrate the segmentation results when the contrast parameter $\lambda$ was varied slightly. 


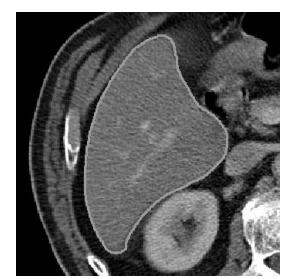

(a) Original man

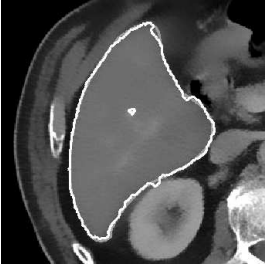

(e) B 16-6-60 best

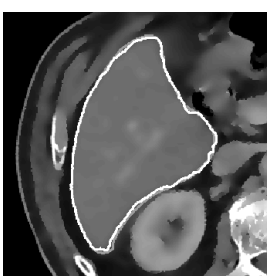

(b) A 50-0.5 best

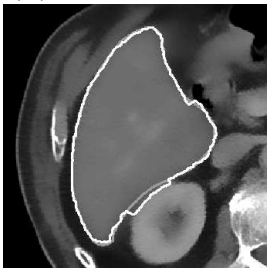

(f) B 16-6-80 neg

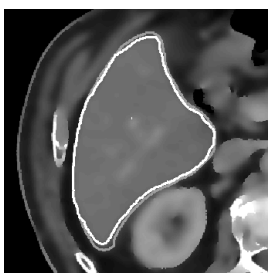

(c) A 50-0.75 neg

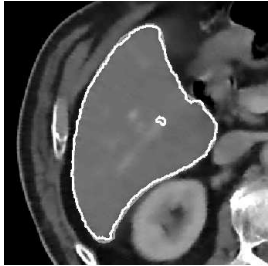

(g) W s-11-11 best

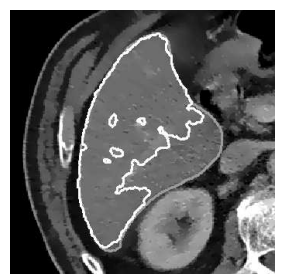

(d) A 50-0.25 neg

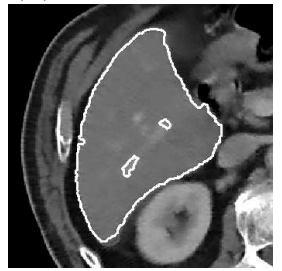

(h) W s-5-6 neg

Fig. 1. GAC segmentation of a liver. Top row: manual segmentation of medical expert and anisotropic diffusion $N_{\text {iter }}-\lambda$. Bottom row: bilateral filtering $m-\sigma_{D}-\sigma_{R}$ and wavelet-based filtering soft-k-N. The manual segmentation (gray) and the results of the automatic segmentation (white) are plotted in the denoised images in (b)-(h): man $=$ manual segementation; best $=$ best segmentation; neg $=$ negative example

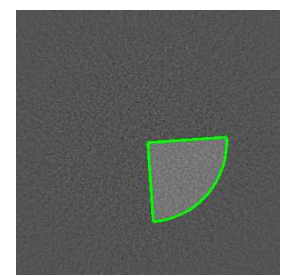

(a) $40 \mathrm{HU}, \sigma_{N}=40$

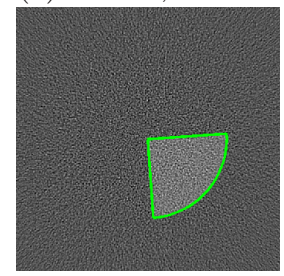

(e) $40 \mathrm{HU}, \sigma_{N}=80$

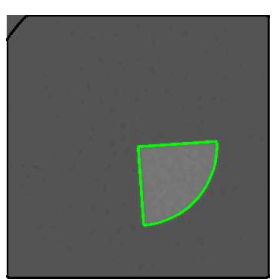

(b) bilateral

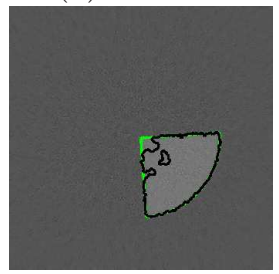

(f) bilateral

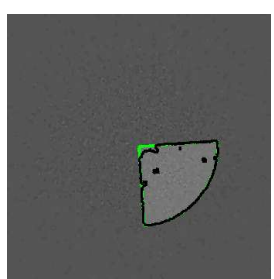

(c) aniso. diffusion

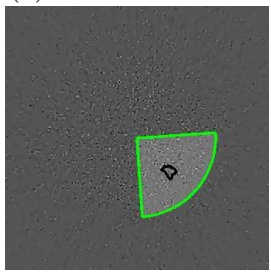

(g) aniso. diffusion

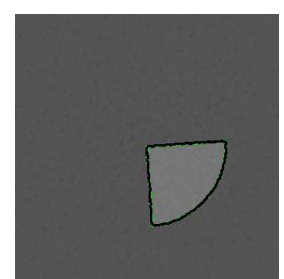

(d) wavelet

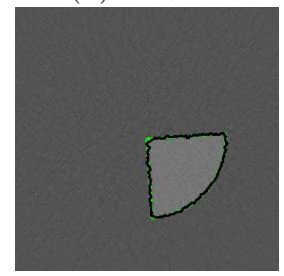

(h) wavelet

Fig. 2. GAC segmentation results: Filtering performed with the same parameter combination on a phantom image with a contrast of $40 \mathrm{HU}$ but different noise $\left(\sigma_{N}\right)$. The ideal contour (gray) and segmentation results (black) are plotted in the denoised images 
Table 1. Best segmentation results for the example of the liver and the spleen

\begin{tabular}{lrrrrrrrrrr}
\hline & \multicolumn{2}{c}{$\begin{array}{c}\text { without } \\
\text { denoising }\end{array}$} & \multicolumn{2}{c}{$\begin{array}{c}\text { best } \\
\text { linear }\end{array}$} & \multicolumn{2}{c}{$\begin{array}{c}\text { best } \\
\text { bilateral }\end{array}$} & \multicolumn{2}{c}{$\begin{array}{c}\text { best } \\
\text { anisotropic }\end{array}$} & \multicolumn{2}{c}{$\begin{array}{c}\text { best } \\
\text { wavelet }\end{array}$} \\
\hline & iter. & s. $\times$ s. & iter. & s. $\times$ s. & iter. & s. $\times$ s. & iter. & s. $\times$ s. & iter. & s. $\times$ s. \\
\hline liver & 290 & 0.08 & 1449 & 0.94 & 794 & 0.98 & 911 & 0.97 & 847 & 0.97 \\
spleen & 155 & 0.53 & 515 & 0.94 & 717 & 0.99 & 460 & 0.98 & 503 & 0.98 \\
\hline
\end{tabular}

An increasing $\lambda$ decreased the slope of the edge and the curve evolution process, starting in the interior of the liver, stopped before the ideal contour was reached. If $\lambda$ was selected too small, the segmentation process stopped after few iterations without covering the whole liver. It can be observed that especially there strong noise could not be removed but was even amplified. The best segmented liver achieved with the wavelet-based filter is shown in Fig. 1(g). It is visible that structures like vessels filled with contrast agent could not be covered during the curve evolution, as during the filtering process even those edges were preserved. If the amount of noise suppression was substantially reduced, (Fig. 1(h)) the outer contour of the liver still matched, however, the holes in the interior increased.

Another observation was that only for the wavelet-based noise reduction method one single parameter combination could be found, leading to good segmentation results for all organs in both datasets. For the anisotropic diffusion and the bilateral filtering even organs of the same dataset could not be segmented without adapting parameters. This is also confirmed by the analysis of simulated phantom images. Figure 2 shows results obtained when for each filter one single parameter set, determined from optimization in real medical data, is applied to images with constant contrast but varying noise level. In contrast to the bilateral filtering and the anisotropic diffusion, the wavelet-based denoising allowed good segmentations for all different noise levels $\left(\sigma_{N} \in\{20,40,60,80\}\right)$.

In Table 1 the best achieved results for two examples are listed. The quality of the segmentation is quantified with the product of sensitivity and specificity, leading to values in the range between 0 and 1 . The larger the value, the better the result of the segmentation. Furthermore, the numbers of iterations used for the segmentation are included. For better comparison the values for the segmentation without any pre-filtering and the best results achieved with a linear Gaussian filtering are shown. The segmentation contours obtained in the liver without pre-filtering, with optimized Gaussian filtering $(\sigma=2)$ and too strong Gaussian filtering $(\sigma=4)$ are shown in Fig. 3 together with the initial segmentation curve. It is obvious that for segmentation a pre-filtering is needed. If too strong linear filtering is performed, the segmentation contour can leak or the precision of the result is reduced. Table 1 shows that with edge-preserving denoising either the number of elapsed iterations could be reduced (example of the liver) or the precision of the segmentation results improved (both examples). 
Fig. 3. Comparison of segmentation results (white) without pre-filtering and with linear Gaussian filtering

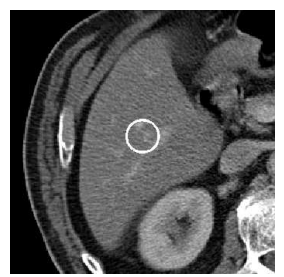

(a) initial curve

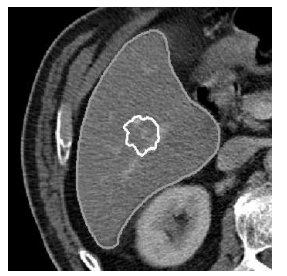

(b) without

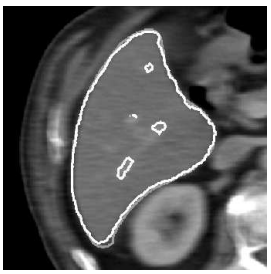

(c) best linear

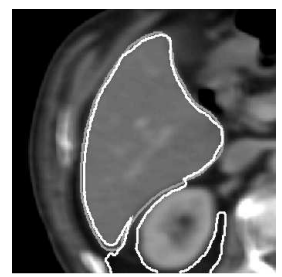

(d) linear negative

\section{Discussion}

The evaluation presented above clearly shows that for the segmentation of organs in medical CT-images a noise-reducing pre-processing step is required. In contrast to linear Gaussian filtering, edge-preserving denoising methods improved the precision of the segmentation contours. Furthermore, the number of elapsed iterations could be decreased for some organs.

By analyzing and comparing different edge-preserving noise reduction methods, it was evident that the parameters of the bilateral filtering and the anisotropic diffusion had to be adapted to each organ in each dataset separately in order to result in precise segmentations. However, for clinical use it is desirable to optimize parameters for the segmentation and its pre-processing once and then used them for all segmentation tasks. In contrast to the bilateral filtering and the anisotropic diffusion, the wavelet-based method allowed the robust and precise segmentation of different organs with varying noise levels with a single parameter combination, as it automatically adapts itself to the CT-specific noise characteristics.

\section{References}

1. Caselles V, Kimmel R, Sapiro G. Geodesic active contours. Int J Comp Vis. 1997;22(1):61-79.

2. Tomasi C, Manduchi R. Bilateral filtering for gray and color images. Proc ICCV. 1998; p. 839-46.

3. Perona P, Malik J. Scale space and edge detection using anisotropic diffusion. IEEE Trans Pattern Anal Mach Intell. 1990;12(7):629-39.

4. Borsdorf A, Raupach R, Hornegger J. Separate CT-reconstruction for orientation and position adaptive wavelet denoising. Proc BVM. 2007; p. 232-6.

5. Borsdorf A, Raupach R, Hornegger J. Wavelet-based noise reduction by identification of correlations. Lect Note Comp Sci. 2006;4174:21-30. 